\title{
INMIGRACIÓN Y MULTICULTURALISMO EDUCATIVO: EL CASO DE LOS ESTUDIANTES DOMINICANOS EN LAS ESCUELAS PUERTORRIQUEÑAS
}

Wilson J. González-Espada

Arkansas Tech University, USA

\section{INMIGRACIÓN DOMINICANA A PUERTO RICO}

Históricamente, el movimiento bidireccional de personas entre la República Dominicana y Puerto Rico siempre ha existido (Pérez-Memen, 1989). Sin embargo, luego de la segunda mitad del siglo XX se ha visto un incremento significativo en Puerto Rico de personas provenientes de la República Dominicana. Específicamente, las grandes olas inmigratorias comienzan en las décadas de los 60 y 70 . La motivación del immigrante en aquella coyuntura histórica era principalmente de corte político.

Más recientemente son mayormente los aspectos económico y de pobreza los que llevan al dominicano a salir de su patria (Martínez-San Miguel, 1998; Ortíz-Negron, 1988). Un ejemplo de la desigualdad económica que impera actualmente en la República Dominicana es el hecho de que la mitad más pobre de los ciudadanos dominicanos recibe menos del $20 \%$ del Producto Bruto Nacional, mientras que las personas pudientes, un $10 \%$ de la población, recibe casi el $40 \%$ del mismo (CIA Factbook, 2004). A partir del año 2000, dos factores son primordialmente responsables de la inmigración de miles de familias dominicanas a Puerto Rico: (1) la desaceleración de la economía norteamericana, país destino de un 84\% de las exportaciones y (2) un aumento en la tasa de desempleo promedio que mantiene a uno de cada cinco dominicanos fuera del mercado laboral formal (CIA Factbook, 2004).

Muchos de los inmigrantes dominicanos llegan a Puerto Rico legalmente a través de visas de trabajo. Contrario a éstos, otros llegan a Puerto Rico con visas de turista y se quedan, o llegan ilegalmente en barcazas luego de cruzar los 130 kilómetros del peligrosísimo Canal de la Mona.

Los "indocumentados" buscan en Puerto Rico una mejor calidad de vida a la vez que proveen económicamente a aquellos familiares que dejaron. Muchos aceptan trabajos de relativa baja paga, aunque el salario mínimo federal norteamericano que aplica a Puerto Rico es mucho más alto que muchos salarios por hora en la República Dominicana. Los inmigrantes dominicanos trabajan principalmente en la construcción, como cocineros, empleadas de la aguja, empleadas domésticas y mecánicos automotrices (Duany, 1988), llenando un espacio importante de la economía puertorriqueña al aceptar trabajos de baja demanda local y aquellos con condiciones de trabajo debajo del promedio.

Debido a su condición económica, los inmigrantes dominicanos se ubican generalmente en las escalas de clase más bajas. Ellos tienden a concentrarse en ciertas áreas urbanas pobres del área metropolitana, que incluye San Juan (Puerto Nuevo, Río Piedras), Bayamón y Caguas. En las últimas décadas, la mayoría de los inmigrantes han establecido sus residencias y negocios en Santurce. En estos centros urbanos, se estima que la población dominicana alcanza el 30\% del total de habitantes (Thomson, 1990). 
Muchos inmigrantes dominicanos ven su estadía en Puerto Rico como una temporera y añoran el retorno a su patria tan pronto como la situación económica mejore allá. Irónicamente, éstos perciben su inmigración como libre, pero involuntaria; es libre porque el inmigrante toma una decisión consciente de viajar, pero es involuntaria porque la persona no desea dejar su patria. Una consecuencia de este hecho es que los inmigrantes dominicanos tratan al máximo de mantener su identidad cultural.

\section{2. ¿CUÁL ES LA DIFERENCIA ENTRE DOMINICANOS Y PUERTORRIQUEÑOS?}

Algunos académicos sugieren que la relación política de naturaleza cuasi-colonial entre los EE.UU. y Puerto Rico (Trías-Monge, 1997), que incluyó un agresivo proceso de "americanización" en la primera mitad del siglo XX y la otorgación de la ciudadanía americana a los puertorriqueños en 1917, puede ser un factor que contribuya a que el puertorriqueño se vea a sí mismo como diferente de otros países de Latinoamérica, incluyendo la República Dominicana (Eliza-Colón, 1989; Negrón de Montilla,1990; Solís, 1994)

Otros académicos proponen que no existe una diferencia real entre dominicanos y puertorriqueños. Ambos grupos comparten el idioma español, una herencia mestiza (mayormente afro-española) y una cultura caribeña. Martínez-San Miguel (1998) va más lejos al sugerir que "dada su raza y cultura, la población dominicana es peligrosamente similar a una mayoría de la población puertorriqueña". Sin embargo, dada la condición de inmigrante del dominicano, muchos puertorriqueños han creado una serie de prejuicios y mitos para crear una "realidad alterna" que los lleva a ver a los dominicanos como significativamente diferentes a los puertorriqueños.

Un ejemplo de esta "realidad alterna" es la discriminación racial a la que comúnmente están expuestos los inmigrantes dominicanos, esto a pesar de que ambos grupos distan de ser el prototipo de la raza caucásica. Los puertorriqueños atribuyen a los dominicanos características similares a aquellas atribuídas a los hispanos y afroamericanos por las personas blancas en Estados Unidos. El domincano se percibe incorrectamente en Puerto Rico como "bruto, ignorante, sucio, más propenso al crimen y a la promiscuidad. Además se le responsabiliza por la sobrepoblación, la decadencia urbana, el tráfico de drogas y otros problemas sociales" (Duany, 1987). Una posible razón por la cual el dominicano se percibe de esta manera es el doble sentido del concepto de ilegalidad en este contexto: dado que los dominicanos son ilegales en Puerto Rico, ellos deben ser responsables de actividades ilegales (Martínez-San Miguel, 1998). Desafortunadamente, los medios de comunicación masiva tienden a perpetuar este estereotipo (Duany, 1987).

Otros estereotipos que muchos puertorriqueños tienen de los dominicanos son (a) que éstos son responsables por el desempleo en Puerto Rico pues "desplazan" a los trabajadores locales y (b) que contaminan la "cultura puertorriqueña." Duany (1988) resume brillantemente la controversia cultural entre puertorriqueños y dominicanos:

"Los indocumentados ilegales se han convertido en el chivo expiatorio de la sociedad puertorriqueña. Es como si los extranjeros cargaran con la culpa de todos nuestros vicios colectivos ... Los inmigrantes dominicanos constituyen uno de los grupos étnicos mas estigmatizados de la Isla [Puerto Rico], quizás porque desempeñan las labores menos prestigiosas y peor pagadas ... Su composición racial-mayormente negros y mulatos-ha dificultado grandemente su integración a la sociedad receptora. Pobres, prietos y extranjeros, los dominicanos son una minoría desventajada en un triple sentido: económico, racial y étnico" (pp. 111-112) 
Nótese la similitud entre este conflicto y aquel entre blancos y personas de color, sobre todo hispanos, en los Estados Unidos de Norteamérica. Esta similitud va aún más allá y puede verse explícitamente en el humor y los chistes de corte racial. En los EE.UU. este tipo de chiste tiende a ser común y se usa como válvula de escape de los sentimiento de odio, ira y resentimiento por parte de algunos sectores de la población norteamericana. Del mismo modo, muchos puertorriqueños conocen chistes de dominicanos en los que éstos se describen como poco inteligentes. El humor, según Martínez-San Miguel (1998) se usa para exagerar las diferencias aparentes entre puertorriqueños y dominicanos y para cubrir las innegables similitudes entre estos dos grupos.

Una pregunta resulta inevitable: ¿Por qué se mantiene una percepción negativa del dominicano a pesar de que la gran mayoría de ellos son personas de provecho en la sociedad puertorriqueña? Parte de la respuesta puede explicarse gracias a la psicología de la percepción selectiva (Broadbent, 1958). Todas y cada una de las personas pasan por un proceso de socialización, mayormente durante la pre-adultez, en el cual aceptamos las premisas básicas de nuestra cultura e internalizamos sus valores, puntos de vista, prejuicios y estereotipos (Banks, 1994). Supongamos que a través de este proceso un puertorriqueño termina creyendo que los dominicanos estan involucrados en activides ilegales. Cada vez que esa persona ve un reportaje sobre un dominicano arrestado por conducta criminal, su creencia se verá reafirmada, ésto a pesar de que se presentan muchos más casos de puertorriqueños arrestados que de dominicanos. La persona inconscientemente ignora aquello que no es relevante a su creencia acerca de los dominicanos. Es muy probable que si esta persona ve un reportaje sobre un dominicano haciendo una buena acción o destacándose positivamente, va a pensar que ese individuo es una excepción a la "regla". Interesantemente, la percepción selectiva no necesita instancias diarias que confirmen las creencias de las personas. Es suficiente que el refuerzo de la creencia suceda de manera intermitente.

La percepción selectiva es muy poderosa y no debe ser subestimada, ya que es responsable de muchas creencias de corte político, social, pseudoscientífico y religioso (Shermer, 1997). Esta percepción aparenta estar directamente relacionada con la manera en que el cerebro procesa informacion en general. Alcock (1995) argumenta que el cerebro humano ha evolucionado por cientos de miles de años para desarrollar creencias, no todas basadas en la realidad, que maximizen las probabilidades de supervivencia:

"Nuestro cerebro y sistema nervioso constituyen una máquina generadora de creencias, un motor que produce creencias independientemente de lo que es real o verdadero y lo que no lo es. Este motor de creencias selecciona información del medioambiente, la moldea y la combina con información memorizada para producir creencias generalmente consistentes con creencias previas. Este sistema es capaz de generar tanto creencias falsas como creencias consistentes con la verdad. Las creencias generadas guiarán acciones futuras y, ya sean verdaderas o falsas, son capaces de ser útiles a la persona que las cree" (p. 1).

La pasada discusión sólo puede llevar al lector a concluir que la inmigración ha creado en

Puerto Rico una sociedad pluralista en la que el dominicano es generalmente visto con malos ojos y es víctima frecuente de opresión. Para lograr una sociedad puertorriqueña más justa y equitativa hacia la población inmigrante es importante reconocer la importancia de una perspectiva educativa multicultural como alternativa de justicia social. 


\section{HACIA UN SISTEMA EDUCATIVO MULTICULTURAL EN PUERTO RICO}

La inmigración de ciudadanos dominicanos a Puerto Rico tiene profundas implicaciones para el sistema educativo local, el cual sirve a miles de estudiantes dominicanos. Estos estudiantes merecen una educación que los ayude a escapar de las garras de la pobreza y que les abra la puerta a mejores oportunidades y acceso. Si usamos como referencia el sistema educativo norteamericano, vemos que Puerto Rico debe moverse hacia un sistema educativo multicultural, sobre todo en áreas donde la presencia de estudiantes dominicanos está más concentrada.

La educación multicultural ha sido investigada extensamente en los EE.UU. durante las pasadas décadas (Atwater, 1994; Banks and McGee-Banks, 1995; González-Espada, 2004; Weinberg, 1977). Nieto (1996) define la educación multicultural de la siguiente manera:

"Es un proceso de reforma educativa [...] que reta y rechaza el racismo y otras formas de discriminación en las escuelas y en la sociedad mientras acepta y afirma el pluralismo (étnico, racial, linguístico, religioso, económico y de género, entre otros) que los estudiantes, sus comunidades y sus maestros representan. La educación multicultural permea el currículo y las estrategias de enseñanza usadas en la escuela, así como las interacciones entre maestros, estudiantes, padres y la escuela como institución" (p. 307).

La educación multicultural se fundamenta en tres pilares (Banks y McGee-Banks, 1997):

- Todos los estudiantes, independientemente de su género, clase social, raza, etnicidad o características culturales, tienen el derecho a recibir iguales oportunidades para aprender.

- La manera en que un sistema educativo está estructurado tiene el potencial de afectar negativamente a aquellos estudiantes que no pertenecen al grupo dominante o cuya características culturales sean diferentes a las del grupo dominante. Por ejemplo, algunas investigaciones sugieren que, en Norteamérica, los estudiantes de color tienen más posibilidades de asistir a escuelas inadecuadas, a ser enseñados por maestros poco cualificados y a dejar la escuela. Estos estudiantes tienen menos probabilidades de graduarse de escuela superior, de continuar estudios postsecundarios o de graduarse de la universidad, comparado con estudiantes del grupo mayoritario (US Department of Education, 2003a, 2003b, 2003c).

- Las creencias y percepciones de maestros, administradores y otros estudiantes con respecto a los grupos minoritarios pueden crear un ambiente escolar hostil y que no promueve ni la igualdad educativa ni la completa inclusión de estos grupos en la sociedad receptora.

A pesar de que existen múltiples referencias al multiculturalismo educativo en las escuelas norteamericanas, projectos de investigación sobre este tema en el contexto puertorriqueño son muy escasos (Laboy-González, 1999; Miranda-Méndez, 2000), lo cual añade aún más relevancia a las sugerencias a presentarse a continuación.

La literatura relacionada al multiculturalismo provee algunas interesantes sugerencias que pueden ser aplicadas al contexto puertorriqueño con el fin de comenzar a reestructurar el ambiente escolar y promover una educacion multicultural en áreas de alta densidad de inmigrantes dominicanos. Para determinar cuál es la verdadera naturaleza del problema de los estudiantes dominicanos en las escuelas puertorriqueñas, es importante establecer proyectos de investigación paralelos que provean información 
más precisa acerca de varios aspectos importantes. Cada uno de esos proyectos debe tener como meta conseguir respuestas satisfactorias a los siguientes interrogantes:

- ¿Cuál es la situación académica, social, cultural y económica de los estudiantes dominicanos en las escuelas? ¿Cuál es la experiencia de estos estudiantes? ¿Qué diferentes puntos de vista pueden aplicarse a este problema? Un esfuerzo que no incluya activamente la voz de aquellos que se pretende ayudar esta destinado al fracaso.

- ¿Específicamente, cuáles son las similitudes y diferencias culturales entre puertorriqueños y dominicanos? ¿Es esa diferencia, si existe, un factor que afecte los estilos de aprendizaje de estos grupos? Investigaciones como la sugerida son relevantes dado que, en Norteamérica, se conoce que los estilos culturales, motivacionales y de aprendizaje de los estudiantes de color difieren de los estilos de enseñanza utilizados con más frecuencia en la escuela (RobinsonShade, 1997).

- ¿Cómo la cultura escolar difiere de la cultura de la comunidad y la cultura de la casa? ¿Cómo esas diferencias pueden minimizarse de modo que los estudiantes dominicanos puedan funcionar comfortablemente en estas tres culturas? Existe evidencia que sugiere que a muchos estudiantes se les hace difícil entender las diferencias entre estas tres culturas, lo cual se traduce en pobre aprovechamiento académico y deserción escolar (Aikenhead, 1996; Phelan, Davidson, and Cao, 1991).

- ¿Existe alguna diferencia entre la actitud de los maestros hacia los estudiantes puertorriqueños en comparación con su actitud hacia los estudiantes dominicanos? ¿Qué factores podrían influenciar esa diferencia? De qué manera se pueden crear oportunidades de desarrollo profesional que atiendan y eliminen actitudes negativas hacia los estudiantes dominicanos? Por ejemplo, la literatura sugiere que los maestros y administradores norteamericanos comúnmente tienen expectativas más bajas hacia los estudiantes pobres y los estudiantes de color (Persell, 1977).

- ¿De qué manera el currículo oficial y el currículo oculto, definido como aquel currículo que no es enseñado formalmente pero que los estudiantes aprenden y se relaciona con la cultural escolar y el contenido académico que se incluye o excluye del curriculo oficial, inciden en las actitudes y el aprovechamiento académico de los estudiantes dominicanos en la escuela? ¿Cómo se puede reformar el currículo a corto, mediano y largo plazo para que presente un contenido más global y relevante para todos los estudiantes? ¿Cómo se pueden integrar al currículo las voces, visiones y perspectivas de diversos grupos en un marco de educación global? Por ejemplo, siempre ha existido una relación estrecha entre Puerto Rico y otras naciones caribeñas, pero esa relación raramente se enfatiza en el currículo escolar. Para lograr una revisión curricular exitosa es esencial la colaboración de académicos dominicanos y caribeños. El que académicos y administradores educativos de Puerto Rico decidan exclusivamente que se debe o no se debe incluir en una revisión curricular multiculturalista puede crear un esfuerzo trunco y limitado.

- Dada la aparente existencia de un prejuicio mutuo entre puertorriqueños y dominicanos, ¿Cuál es la mejor manera de confrontar y reducir los prejuicios culturales entre ambos grupos en el 
contexto escolar? Debido a que cada grupo se socializa en un marco diferente, no es común que un grupo conozca las creencias, valores y cultura del otro grupo y las compare con aquellas que el grupo ha creado. Conocer las culturas de diferentes grupos ayuda a los estudiantes a reducir los estereotipos y a desarrollar actitudes positivas hacia éstos (Banks, 1994).

- ¿Cómo los programas de preparación de maestros atienden la educación multicultural? ¿Es ésta una meta importante? ¿Se incluyen experiencias multiculturales cuando los estudiantes hacen práctica docente? Qué cambios en estos programas pueden contribuir a reducir los prejuicios de los futuros maestros?

- ¿Existe un diálogo genuino y responsable entre los funcionarios a cargo del sistema educativo de Puerto Rico y la comunidad dominicana radicada en la Isla? Específicamente cómo los padres de los estudiantes dominicanos pueden colaborar con los maestros en la planificación, enseñanza y evaluación de éstos estudiantes? La influencia de los padres siempre han sido importantes para la educación de sus hijos. Comer (1988) sugiere que el rol de los padres es más importante aún para aquellos estudiantes de bajos recursos económicos y minorías.

- ¿Cuáles son los fundamentos filosóficos del currículo puertorriqueño contemporáneo? ¿Son estos fundamentos consistentes con los hallazgos más recientes en la educación? Desde el punto de vista filosófico, futuras revisiones curriculares deben enfatizar que el conocimiento es una construcción social que refleja las perspectivas, experiencias y valores de la cultura que los crea, lo cual implica que diferentes culturas poseen conocimientos igualmente válidos pero no necesariamente consistentes entre sí. (Sleeter and Grant, 1987). Además, este nuevo currículo debe enfatizar el aspecto transformativo en el que la estructura curricular cambia completamente para dar a los estudiantes la oportunidad de ver datos, eventos, etc. desde múltiples puntos de vista. Crear un currículo parchado con un enfoque contributivo (en el que se incluyen de manera superficial y aislada algunos eventos históricos dominicanos y caribeños en el currículo) o un enfoque aditivo (en el que se incluyen en el currículo contenido y discusiones multiculturales sin cambiar el enfoque monocultural del mismo) no debe ser una solución a largo plazo (Banks, 1988)

\section{CONCLUSIÓN}

Ha sido una meta de este artículo proveer al lector una discusión breve de las raíces históricas de la inmigración de dominicanos hacia Puerto Rico y como la misma propende a la creación de estereotipos que crean un ambiente hostil hacia los estudiantes dominicanos en las escuelas locales. Además se argumenta a favor de extender algunos de los hallazgos de la literatura relacionada con la educación multicultural al contexto puertorriqueño con el fin de comenzar la búsqueda de información y, eventualmente, de soluciones que mejoren el aprovechamiento académico de todos los estudiantes.

Luego de la pasada discusión resulta obvio que no existe una respuesta clara y de fácil implementación al problema de educar adecuadamente a todos los niños en una sociedad pluralista donde las diferencias étnicas son sólo una de las muchas variables que afectan el aprovechamiento académico de los alumnos. El primer paso en la dirección correcta debe ser una exploración profunda y objetiva del tema 
desde una perspectiva teórica aceptada, como el multiculturalismo, y desde una perspectiva práctica y experiencial, protagonizadas por las voces de la comunidad dominicana en Puerto Rico y por los componentes del sistema educativo puertorriqueño.

\section{BIBLIOGRAFÍA}

AIKENHEAD, G.S. (1996). Border crossing: Culture, school science, assimilation of students. En D.A. Roberts \& L. Östman (Eds.), The multiple meanings of a school subject: Essays on science and the school curriculum. New York, NY: Teachers College Press.

ALCOCK, J. (1995). "The belief engine." Skeptical Inquirer, 19(3), 255-263.

ATWATER, M. M. (1994). Research on cultural diversity in the classroom. En D. L. Gabel (ed.), Handbook of research on science teaching and learning. New York, NY: Macmillan, pp. 558-576.

BANKS, J. A., and McGeE-BANKs, C. A. (1997). Multicultural education: Issues and perspectives, $3^{\text {rd }}$ ed. Boston, MA: Allyn and Bacon.

BANKS, J. A. and MCGEE-BANKS, C. A. (1995). Handbook of research on multicultural education. New York, NY: Macmillan.

BANKS, J. A. (1994). An introduction to multicultural education. Boston, M.A:

Allyn and Bacon.

BANKS, J. A. (1988). "Approaches to multicultural curriculum reform."Multicultural Leader, 1(2), 1-3.

CIA FACTBOOK (2004). Dominican Republic. <http://www.cia.gov/cia/publications/ factbook/geos/dr.html> [Consulta: feb. 2005]

COMER, J. P. (1988). "Educating poor minority children”. Scientific American, 259(5),

$42-48$.

DuANY, J. (1988). "Los dominicanos indocumentados en Santurce: Su inserción a la socieded puertorriqueña." Punto y Coma, 1(1), 105-115.

DUANY, 1987. Los indocumentados dominicanos y la opinión pública puertorriqueña. Conferencia presentada en el Centro Católico Universitario, Universidad de Puerto Rico, Río Piedras.

ELIZA-Colón, S. M. (1989). "Colonialism and education in Puerto Rico: Appraisal of the public schools during the Commonwealth period 1952-1986." Dissertation Abstracts International, 51(04), 1139A. (University Microfilms No. AAl90-17364).

GonZÁlEZ-ESPADA, W. J. (2004). "Multicultural education: Helping all students succeed in science." Electronic Journal of Literacy through Science, 3(12), 1-14, <http://sweeneyhall.sjsu.edu/ejlts/> [Consulta: feb. 2005]

LABOY-GonZÁlEZ, B. (1999). Estudio comparativo de las necesidades de estudiantes de escuela intermedia, puertorriqueños y dominicanos: Implicaciones para el consejero escolar.

Universidad de Puerto Rico, Recinto de Río Piedras. Tesis no publicada. 
Martínez-San Miguel, Y. (1998). De ilegales e indocumentados. Representaciones culturales de la migración dominicana en Puerto Rico. Revista de Ciencias Sociales/Nueva Época, 4, 147-173.

MiRANDA-MÉndeZ, A. (2000). Impacto educativo y etnográfico de la inmigración dominicana en Puerto Rico desde 1970-2000: Perspectivas futuras. Universidad de Puerto Rico, Recinto de Río Piedras. Tesis no publicada.

negrón De Montilla, A. (1990). La americanización de Puerto Rico y el sistema de instrucción pública 1900-1930. Río Piedras, Puerto Rico: Editorial de la Universidad de Puerto Rico.

NIETO, S. (1996). Affirming diversity: The sociopolitical context of multicultural education. White Plains, NY: Longman Publishers.

Ortíz-Negrón, L. L. (1988). "En torno a la migración dominicana en Puerto Rico." Revista de Ciencias Sociales, 27(3-4), 147-154.

PercelL, C. H. (1977). Education and inequality: A theoretical and empirical synthesis. New York, NY: Free Press.

Pérez-Memem, F. (1989). Panorama histórico de las emigraciones dominicanas a Puerto Rico. En J. E. Hernández-Cruz, Los immigrantes indocumentados dominicanos en Puerto Rico: Realidades y mitos. San Germán, Puerto Rico. Centro de Publicaciones de la Universidad Interamericana.

Phelan, P., Davidson, A., \& Cao, H. (1991). Students' multiple worlds: Negotiating the boundaries of family, peer, and school cultures. Anthropology and Education Quarterly, 22(3), 224-250.

RoBinson-SHADE, B. J. (1997). Culture, style, and the educative process, $2^{\text {nd }}$ ed. Springfield, IL: C. C. Thomas.

SHERMER, M. (1997). Why people believe weird things: Pseudoscience, superstition, and other confusions of our time. New York, NY: W.H. Freeman.

Sleeter, C. E., and Grant, C. A. (1987). "An analysis of multicultural education in the United States." Harvard Educational Review, 57(4), 421-444.

SoLís, J. (1994). Public school reform in Puerto Rico: Sustaining colonial models of development. Wesport, CT: Greenwood Press.

THOMSON, L. (1990). La migración en una semi-periferia incipiente: Aspectos económicos y socio-culturales de la migración en Puerto Rico. En J. Duany (ed.), Los dominicanos en Puerto Rico: Migración en la semi-periferia. Río Piedras, PR: Ediciones Huracán.

TRíAs-Monge, J. (1997). Puerto Rico: The Trials of the Oldest Colony in the World.

New Haven: Yale University Press.

U.S. Department of Education (2003a). From risk to opportunity: Fulfilling the educational needs of Hispanic Americans in the 21st century. Washington, DC: President's Advisory Commission on Educational Excellence for Hispanic American.

U.S. Department OF Education (2003b). National Center for Education Statistics: Status and trends in the education of Blacks, by Kathryn Hoffman and Charmaine Llagas. Project Officer: Thomas D. Snyder. Washington, DC (NCES 2003-034) 
U.S. Department of Education (2003c). National Center for Education Statistics: Status and trends in the education of Hispanics, by Charmaine Llagas. Project Officer: Thomas D. Snyder. Washington, DC (NCES 2003-008).

WeINBERG, M. (1977). A history of race and education in the United States. Cambridge, MA: Cambridge University Press. 


\title{
Contactar
}

Revista lberoamericana de Educación

\author{
Principal OEI
}

